\title{
Current status of stereotactic body radiotherapy for the treatment of hepatocellular carcinoma
}

\author{
Jongmoo Park ${ }^{1}$, Jae Won Park ${ }^{2}$, Min Kyu Kang ${ }^{1}$ \\ ${ }^{1}$ Department of Radiation Oncology, School of Medicine, Kyungpook National University, Daegu, Korea \\ ${ }^{2}$ Department of Radiation Oncology, Yeungnam University College of Medicine, Daegu, Korea
}

Received: July 3, 2019

Revised: July 22, 2019

Accepted: July 30, 2019

Corresponding author:

Min Kyu Kang

Department of Radiation Oncology,

Kyungpook National University

Chilgok Hospital, 807, Hoguk-ro,

Buk-gu, Daegu 41404, Korea

Tel: +82-53-200-2653

Fax: +82-53-200-2029

E-mail: mkkang@knu.ac.kr
Stereotactic body radiotherapy (SBRT) is an advanced form of radiotherapy (RT) with a growing interest on its application in the treatment of hepatocellular carcinoma (HCC). It can deliver ablative radiation doses to tumors in a few fractions without excessive doses to normal tissues, with the help of advanced modern RT and imaging technologies. Currently, SBRT is recommended as an alternative to curative treatments, such as surgery and radiofrequency ablation. This review discusses the current status of SBRT to aid in the decision making on how it is incorporated into the HCC management.

Keywords: Hepatocellular carcinoma; Image-guided radiotherapy; Radiotherapy; Stereotactic body radiotherapy

\section{Introduction}

Liver cancer is the sixth most common cancer and the second leading cause of cancer-related mortality in Korea [1]. Hepatocellular carcinoma (HCC) is the major histologic type of primary liver cancers, accounting for $70-85 \%$ [2], and has the highest mortality rate among major cancers in Korea [3]. Current treatment guidelines for HCC recommend surgical resection, liver transplantation, and radiofrequency ablation (RFA) as standard treatment options for small HCCs $[4,5]$. However, hepatic resection is possible in only $<30 \%$ of patients due to multifocal disease, unfavorable location, or inadequate functional hepatic reserve, and liver transplantation is associated with many hurdles such as donor availability [6]. RFA is not always safe or effective depending on the tumor size or locations [7].

Stereotactic body radiotherapy (SBRT), or stereotactic ablative radiotherapy, is an advanced form of radiotherapy (RT) that can deliver ablative radiation doses to tumors in a few fractions without excessive doses to normal tissues with the help of advanced modern RT and imaging technologies [8]. Several case series on SBRT have reported high local control rates of over $80 \%$ [9]. Current treatment guidelines for HCC recommend SBRT when other liver-directed therapies have failed or are contraindicated $[4,5,10]$. In addition, it is tried to be combined with other locoregional or systemic therapies.

However, radiation-induced complications in the liver and gastrointestinal (GI) tract prevent the use of SBRT. Patients with HCC often have cirrhotic liver with poor baseline liver function or less recovery ability, which could be related to a higher incidence of radiation-induced liver disease (RILD). The GI tract near the tumors often prevents high-dose irradiation from being delivered to the tumors. Respiratory movement of the liver containing tumors prevents the safe use of SBRT. For these reasons, various efforts have been implemented to understand the risk of toxicities and determine solutions.

This review discusses the current status of SBRT for HCCs in 
terms of technical and clinical aspects to help in the decision making of using SBRT while treating patients with HCC.

\section{Radiotherapeutic considerations}

\section{Process of stereotactic body radiotherapy}

The first step of treatment planning is acquisition of planning computed tomography (CT) images in the treatment position. Immobilization devices, such as stereotactic body frame and vacuum cushions, are usually used to improve the positional reproducibility and reduce the possible movement during the long treatment time of SBRT. Four-dimensional CT (4D-CT) scan with intravenous contrast media is usually used to identify tumor and normal organ movements during respiration. Depending on methods used for tumor localization and respiratory control during treatment, planning $\mathrm{CT}$ scans can be performed in various settings: free breathing or breath-hold with or without abdominal compression.

After transferring CT images to the RT treatment planning system, target volumes and normal organs are delineated. Gross tumor volume (GTV) is defined as the tumor(s) seen on imaging studies. In cases of invisible tumor(s) or unclear tumor margin on planning CT images, rigid or deformable registration with diagnostic CT or magnetic resonance imaging (MRI) can be used. The clinical target volume for SBRT is usually the same as for GTV, even though microscopic tumor extension might exist up to several millimeters. The internal target volume (ITV) is the sum of GTVs in all respiratory phases (or some predefined phases for respiratory gating). Planning target volume (PTV) is generated by adding a margin of about $5 \mathrm{~mm}$ (up to $10 \mathrm{~mm}$ ) to ITV [11]. Thereafter, treatment plans are generated while considering tolerance doses of normal organs, especially for the normal liver and GI tract. Typically, 40-50 Gy in 3-5 fractions is prescribed to encompass the edge of PTV [11-14].

Immediately before treatment, the patient and tumor position are verified with orthogonal radiographs or cone-beam CT scans using various surrogate markers, such as the diaphragm, surgical clips, residual lipiodol after transarterial chemoembolization (TACE) or fiducial markers inserted a few days before planning CT. Recently, the MRI device integrated into the RT machine can be used for real-time tumor tracking during treatment. Finally, treatment starts with respiratory motion management such as active breathing control [15], abdominal compression [16], respiratory gating, and real-time tumor tracking. Respiratory gating is a technique that can treat tumors only at predefined respiratory phases. Tumor-tracking technique is thought to be an ideal method to treat moving targets, which can treat the tumors with the smallest PTV compared to other methods. With the proper use of these techniques, SBRT-related toxicities in the liver and GI tract can be minimized while ensuring enough doses to tumors.

Various imaging tools described above are used to maximize the accuracy and precision throughout the whole process, which is called image-guided radiotherapy (IGRT). The technical aspects of RT for HCC are discussed in detail elsewhere [17,18]. Fig. 1 shows an example of SBRT using tracking intensity-modulated beams.

\section{Tumor dose}

Radiation oncologists have used various dose fractionation schedules and investigated the impact of total dose and tumor size. For small tumors of $<3 \mathrm{~cm}$ (or $<5 \mathrm{~cm}$ ), a high local control of $>90 \%$ has been achieved with a biologically effective dose (BED) of $\geq 100 \mathrm{~Gy}_{10}$ (e.g., $50 \mathrm{~Gy}$ in 5 fractions or 45 Gy in 3 fractions), which is considered as an ablative dose $[12,19,20]$. BED, using a conventional linear-quadratic model assuming $\alpha / \beta$ of $10 \mathrm{~Gy}$, was calculated as follows: $\mathrm{BED}=\mathrm{nd}(1+\mathrm{d} /[\alpha / \beta])$, where $\mathrm{n}$ is the number of fractions and $d$ is the fraction size. In a retrospective study that escalated the radiation dose from 45 Gy in 3 fractions to 60 Gy in 3 fractions for HCCs of $<3 \mathrm{~cm}$, no difference in local control was found between the 2 dose groups [20]. In contrast, several studies have reported lower local control rates in larger tumors $[12,19,21]$. Although Jang et al. [21] revealed that large tumors needed a higher radiation dose for the same tumor control probability of $90 \%$ as small tumors (51.1 Gy in 3 fractions for tumors of $\leq 5 \mathrm{~cm}$ vs. $62.1 \mathrm{~Gy}$ in 3 fractions for tumors of $>5-7 \mathrm{~cm}$ ), higher radiation doses would inevitably increase the liver dose related to liver toxicity. Sanuki et al. [13] achieved a high 3-year local control rate of $91 \%$ with relatively low doses (40 Gy in 5 fractions in Child-Pugh [CP] A and 35 Gy in 5 fractions in CP B). A pooled analysis of 25 studies on SBRT revealed that overall survival was not different according to radiation doses (equivalent dose in 2 Gy per fractions [EQD2] using the $\alpha / \beta$ ratio of $10 \mathrm{~Gy}$ : < $80 \mathrm{~Gy}$ vs. $\geq 80 \mathrm{~Gy}$ ), where all available median EQD2 estimates ranged from 47.9 Gy to 100 Gy with a median of $83.3 \mathrm{~Gy}$ (equal to 100 $\mathrm{Gy}_{10}$ in BED) [9]. Therefore, considering that higher radiation dose and/or larger tumor volume could increase hepatic and/or GI toxicities, the risk adaptive dose-fractionation regimens would be useful to balance both tumor control and toxicities, especially in patients with poor liver function.

\section{Charged particle therapy}

Charged particles such as proton or carbon ions have a unique beam profile known as Bragg peak, which can prevent irradiation of normal tissues beyond the tumor. Treatment outcomes in patients with HCC treated with proton or carbon ions are promis- 


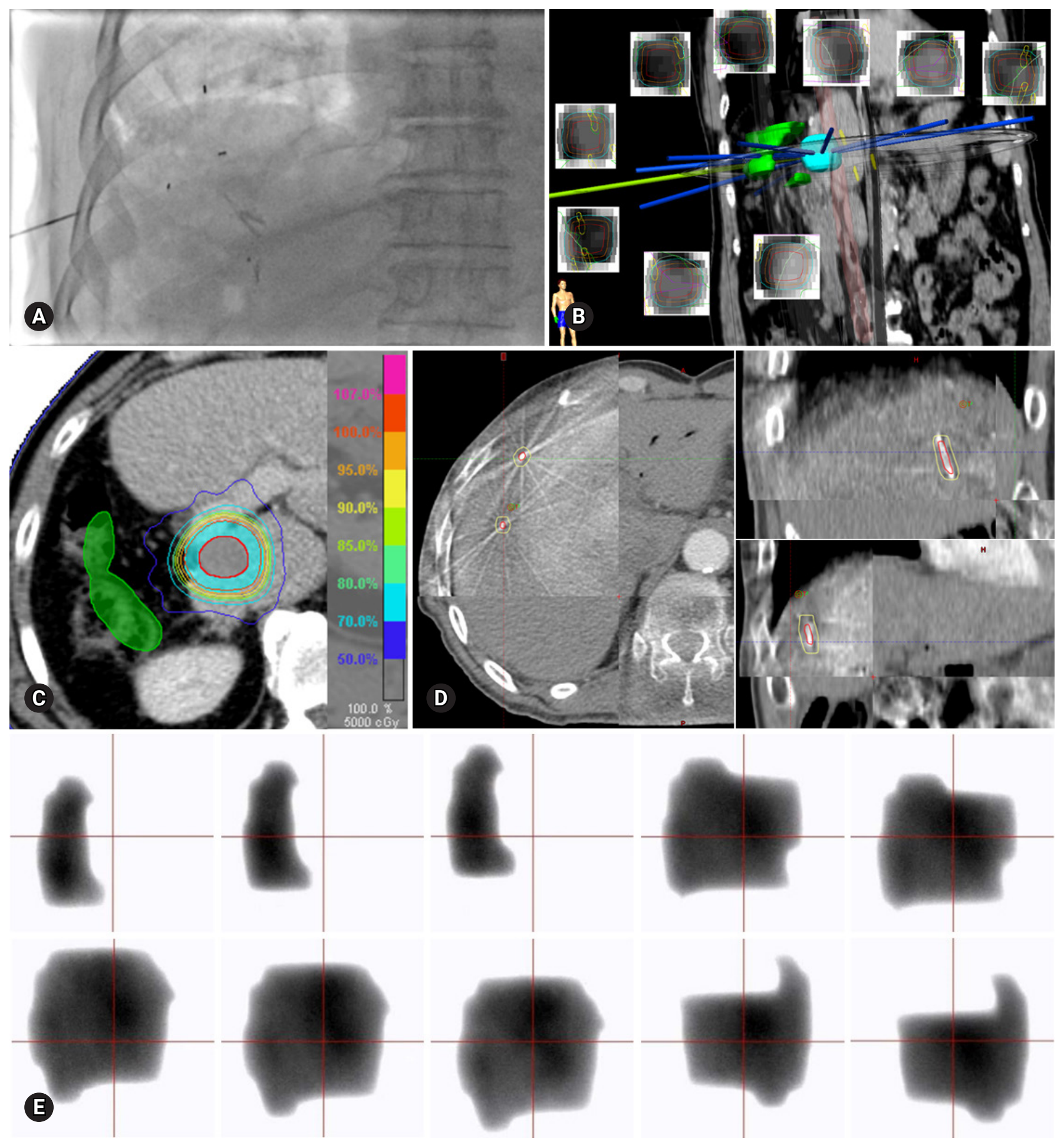

Fig. 1. An example of stereotactic body radiotherapy using tracking intensity-modulated beams for a 20-mm-sized lesion in segment 1 that occurred after right hepatectomy and 5 times of radiofrequency ablation. (A) Gold fiducial markers are inserted before the simulation for patient/tumor position verification and real-time tumor tracking. $(B, C)$ Target volumes and normal tissues are delineated in breath-hold images, and then treatment plan using 9 intensity-modulated beams is generated (gross tumor volume in red, planning target volume in cyan, and the intestine in green). (D) In the treatment room, locations of fiducial markers are verified using cone-beam computed tomography. (E) The treatment beams are delivered while monitoring the movement of the fiducial markers in real time. Electronic portal imaging device shows the movement of each beamlet during tracking treatment. Images in $B, C$, and $E$ are from this patient, and images in $A$ and $D$ are from other patients for demonstration. 
ing with high local control rates of $87-90 \%$ at 3 years [22,23]. Based on dosimetric studies comparing the proton and photon, proton SBRT was preferred for larger tumors in terms of liver toxicity and could meet liver dose constraints even when photon SBRT could not $[24,25]$. Despite the limited number of particle therapy facilities, charged particle therapy is a promising modality for the treatment of HCC.

\section{Clinical considerations}

\section{Clinical outcomes of stereotactic body radiotherapy}

Many institutions have reported their clinical outcomes after SBRT for HCC after the first report by Blomgren et al. [26]. Even though dose schedules and patient/tumor characteristics were heterogeneous, clinical outcomes were promising in terms of tumor control and toxicities. Recently, Rim et al. [9] conducted a meta-analysis on the results of 32 studies, including 1,950 patients, published in between 2010 and 2018. The median proportion of CP A was $82.3 \%$ (range, 47.9-100\%). The median of the median tumor sizes was $3.3 \mathrm{~cm}$ (range, 1.6-8.6 cm). The median of the median EQD2 estimates was $83.3 \mathrm{~Gy}$ (range, 48.0-114.8 Gy). Pooled 1-, 2-, and 3-year overall survival rates were $72.6 \%$ (95\% confidence interval [CI], 65.7-78.6), 57.8\% (95\% CI, 50.9-64.4), and 48.3\% (95\% CI, 40.3-56.5), respectively. Pooled 1-, 2-, and 3-year local control rates were $85.7 \%$ (95\% CI, 80.190.0), 83.6\% (95\% CI, 77.4-88.3), and 83.9\% (95\% CI, 77.688.6 ), respectively. Larger tumor size of $\geq 5 \mathrm{~cm}$ was a significant factor for worse local control and overall survival rates. Hepatic and GI complication rates of grade $\geq 3$ were $4.7 \%$ (95\% CI, 3.46.5 ) and 3.9\% (95\% CI, 2.6-5.6), respectively. Overall, these results imply that SBRT is a safe and effective local ablative therapy.

\section{Selection of local modalities}

Tumors unsuitable for ablation or surgical resection or with failed treatments have usually been treated with SBRT; however, deciding whether a patient is a proper candidate for SBRT or which is the best modality is difficult. Data below would help the multidisciplinary team to recommend SBRT for a given patient, even though the evidence level is low.

Overall local control rates of SBRT and RFA have been reported to be comparably high $[27,28]$. SBRT was notably achieved a higher local control rate than RFA for tumors of $\geq 2 \mathrm{~cm}$ in Wahl et al.s study [28], which may be related to the better radiation dose coverage of SBRT and limited heat transfer to long distances from the heat source of RFA. In addition, tumor control was not influenced by the tumor size after SBRT in two studies with a median tumor size of $2.2 \mathrm{~cm}$ (range, $0-10.0 \mathrm{~cm}$ ) and $7.2 \mathrm{~cm}$ (range, 1.4-23.1 cm) [28,29], which implies that SBRT could be a preferred treatment option for large tumors.

RFA is less effective or contraindicated in the following situations: tumors close to the major vessels (due to the heat sink effect); tumors abutting the diaphragm (due to the risk of diaphragmatic injury); tumors on the liver capsule (due to the risk of rupture or track seeding); centrally located tumors (due to the risk of bile duct injury); and invisible tumors on ultrasonography [30]. Instead, SBRT is less influenced by tumor location, except its distance from the GI tract.

An important difference between RFA and SBRT is repeatability. In contrast to RFA, repeated use of SBRT is limited for new HCC lesions due to the decreased liver function after SBRT [31]. Considering that frequent intrahepatic recurrences of $\mathrm{HCC}$ require repeated treatments, SBRT might be spared for lesions unsuitable for other liver-directed treatments. In contrast, Lee et al. [32] reported the treatment outcomes after a repeated SBRT for recurrent HCCs with a median tumor size of $1.7 \mathrm{~cm}$ (interquartile range, 1.4-2.2 cm) in 85 patients. The 3-year local control rates were not different between the first and second SBRT ( $94.9 \%$ vs. $90.4 \%, p=0.667)$. None of the 73 patients with CP A experienced RILD after the second SBRT, whereas 2 of the 12 patients with CP $\mathrm{B}$ experienced irreversible liver function deterioration. Although the use of repeated SBRT has been limited so far, SBRT can be reapplied to highly selected patients with good liver function.

The superiority in the survival aspect between SBRT and other treatments has been controversial. RFA showed superior survival compared to SBRT in a study based on a large number of patients from the National Cancer Database [33]. When comparing SBRT with surgical resection, survival was comparable or superior in the surgery group, depending on the studies $[34,35]$. However, a firm conclusion cannot be made because of both the retrospective design and potential selection bias in these studies.

\section{Toxicities after stereotactic body radiotherapy}

\section{Liver}

Liver toxicities, or RILD, is the most important dose-limiting factor for the treatment of liver tumors [36]. Patients with HCC are more vulnerable to the development RILD, because HCC frequently occurs in a cirrhotic liver susceptible to radiation injury. Non-classic RILD (elevation of liver transaminases or decline of CP score) is usually reported after SBRT, rather than classic RILD (anicteric hepatomegaly and ascites or elevation of alkaline phosphatase). The RILD rate of grade $\geq 3$ was as low as $4.7 \%$ ( $95 \%$ CI, 3.4-6.5) in a pooled analysis of 23 studies, which was 
generally transient [9]. Since worse baseline liver function is correlated with higher risk of severe RILD [13,37-40], caution is warranted in deciding the treatment and SBRT planning in patients with poor liver function. Although no definite guidelines were available for liver constraints, the following guidelines have been used: limiting SBRT to CP A or B7 patients [5]; adjusting prescription dose using the normal tissue complication probability of the liver [39]; limiting the normal liver dose (e.g., mean normal liver dose: < 13-18 Gy in 3-6 fractions for CP A vs. <6 Gy in 4-6 fractions for CP B) [36]; or sparing the critical residual liver volume (e.g., $\geq 700 \mathrm{~mL}$ of normal liver receives $\leq 15 \mathrm{~Gy}$ in 3-5 fractions) [36].

\section{Gastrointestinal tract}

Because tolerance doses of luminal structures such as the esophagus, stomach, or intestine are much lower than ablative doses used for SBRT, special concerns are required for lesions close to luminal structures. In one case series, all 5 patients who experienced grade 3 or 4 GI toxicities had lesions within $0-0.4 \mathrm{~cm}$ from the GI tract [21]. Radiation oncologists generally recommend SBRT for lesions with enough distance from GI tract (e.g., >1-2 cm) $[14,41]$. To overcome this limitation, various techniques are attempted, including 4D-CT, abdominal compression, gated RT, tracking RT, or intensity-modulated beam. Real-time tumor tracking, using fiducial markers (Fig. 1A) or MRI images, is the current most advanced method used to avoid the GI tract close to tumors. Fig. 1 shows an example of a patient undergoing follow-up without GI complication at 33 months after receiving tracking SBRT for a tumor at $5 \mathrm{~mm}$ from the bowel. Tolerance doses of luminal structures are discussed in detail elsewhere [42-45].

\section{Bile duct}

Biliary stricture has been occasionally reported at the rate of $0-3 \%$ [12,28,32,46-48]. Eriguchi et al. [49] found biliary stenosis in 2 out of 50 patients irradiated with over 20 Gy to the central biliary system; 1 patient experienced biliary stricture at the area irradiated with $>80$ Gy after the second SBRT and the stenotic site of the other patient was outside the area irradiated with $>20 \mathrm{~Gy}$. In addition, even in a study that investigated the relationship between the central hepatobiliary tract dose and hepatobiliary toxicity rate (not only for biliary tract complications), no biliary stricture was observed in 20 patients with HCC unlike the intrahepatic cholangiocarcinoma with high rate of biliary stricture (38.8\% of 26 patients) [50]. Therefore, SBRT seems to be a feasible modality for centrally located tumors unsuitable for other treatments.

\section{Additional indications of stereotactic body radiotherapy}

\section{As a bridge therapy}

Bridge therapies can be used to prevent tumor progression while waiting for transplantation or to downstage tumors into the Milan criteria. SBRT as a bridge therapy has also been reported as safe and effective. O'Connor et al. [51] and Andolino et al. [52] reported that SBRT did not increase surgical complications. Sapisochin et al. [53] revealed that drop-out, post-transplant survival, and HCC recurrence rates after liver transplantation were similar among SBRT, TACE, and RFA. Mohamed et al. [54] showed that acute toxicities of SBRT and yttrium-90 radioembolization were lower than those of TACE or RFA. These results suggest that $\mathrm{SBRT}$ is a viable option as bridge to transplantation.

\section{In advanced hepatocellular carcinoma}

HCC with portal vein tumor thrombosis (PVTT) has poor prognosis; however, the standard treatment strategy remains to be established. RT has been used to restore the portal flow and facilitate subsequent treatments such as surgery or TACE. The response rate was $39-57 \%$ with conventional RT using a small fraction size of 1.8-3 Gy, which requires a long treatment period (e.g., 45-50 Gy in 25 fractions over 5 weeks) [55]. Advanced RT techniques such as intensity-modulated RT or IGRT facilitate giving higher radiation doses without increasing GI toxicities, with which higher tumor response and better survival would be expected [56]. Although advanced tumor characteristics related to PVTT, poor liver function, or close distance to GI tract often limit the use of SBRT for PVTT [57], recent studies have reported high PVTT response rates (70-76\%) after SBRT using relatively low dose regimens (e.g., 40 Gy in 5-6 fractions over 1-2 weeks) [58-60]. Therefore, SBRT, which ends in a short treatment period, is worth of future investigation for the treatment of PVTT, especially in combination with recently developed systemic agents.

Large or multiple HCCs not suitable for curative treatments are often treated with TACE with or without RT $[4,5]$. A meta-analysis by Meng at al. [61], comparing TACE plus RT to TACE alone, revealed that the adding RT to TACE improved the tumor response and survival, where small doses per fraction or lower total doses were usually used for fear of toxicities in the liver and GI tract. As SBRT has a higher capability of sparing normal tissues as compared to RT techniques used before, SBRT could be assumed to increase the clinical outcomes when combined with TACE for large HCCs. Jacob et al. [62] reported that TACE plus SBRT (45 Gy in 3 fractions) achieved better local 
control and survival rates than TACE alone in HCCs of $\geq 3 \mathrm{~cm}$. Paik et al. [63] confirmed the comparable survival outcomes among the three treatment groups (complete TACE alone, incomplete TACE followed by curative treatments, and incomplete TACE followed by SBRT [46-60 Gy in 3-5 fractions]). These results imply that SBRT is an effective adjuvant treatment for HCCs showing an incomplete response to TACE.

\section{In combination with systemic therapies}

Despite the high local control rates of SBRT, there is a demand to combine SBRT with systemic agents due to frequent intrahepatic or extrahepatic recurrences after SBRT. Sorafenib, a multikinase inhibitor, was attempted to be combined with SBRT based on improved overall survival in patients with advanced HCC treated with sorafenib (vs. best supportive care group) in SHARP phase III trial [64] and in vitro and in vivo radiosensitizing effect of sorafenib in HCC cell lines [65]. However, the addition of sorafenib to SBRT is not currently recommended due to increased risk of GI toxicities [66,67].

Recently, immunotherapy has been successfully employed for the treatment of solid tumors such as melanoma and non-small cell lung cancer, and the combination of immunotherapy with RT is an active field of clinical investigation [68]. SBRT using a high radiation dose per fraction is known to have an immunomodulatory effect, which can potentially improve tumor response and survival outcomes of immunotherapy [69]. Notably, abscopal effect, or tumor regression outside the RT field, has been increasingly reported when RT was combined with immunotherapy, even though it was a rare phenomenon before the era of cancer immunotherapy. Mechanisms of abscopal effect, related to systemic effect of radiation, are also being revealed [70,71]. In 2017 and 2018, 2 immune checkpoint antibodies, nivolumab and pembrolizumab, have been approved to be used as a second-line therapy for HCC by the Food and Drug Administration. Preclinical data showed that the combination of programmed death ligand 1 blockade and RT significantly suppressed the tumor growth in a murine HCC model compared with single treatments [72]. And, there are several ongoing phase I or II trials combining immunotherapy and SBRT for patients with HCC (NCT03203304, NCT03316872, NCT03482102, and NCT03817736). With this new strategy, the prognosis of both early and advanced HCCs is anticipated to improve via enhanced local and systemic effects.

\section{Conclusion}

Current evidences support the use of SBRT as an alternative or complement to curative treatments based on its high local con-
Table 1. Clinical uses of stereotactic body radiotherapy for hepatocellular carcinoma

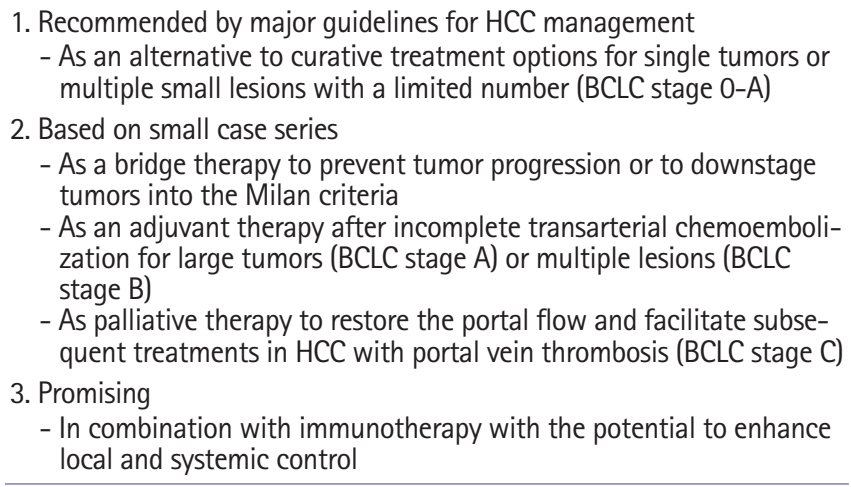

HCC, hepatocelluar carcinoma; BCLC, Barcelona Clinic Liver Cancer.

trol rates and low toxicity profiles. There is also an emerging interest in combining SBRT with other locoregional or systemic therapies to potentially maximize treatment outcomes. Clinical uses of SBRT are summarized in Table 1. However, optimal dosefractionation regimens balancing tumor control and toxicities and methods to overcome the current limitations of SBRT should be determined. Given that clinical presentations of patients with HCC are significantly heterogeneous, a multidisciplinary team approach could determine the best candidates who would benefit from SBRT with or without combined treatments.

\section{Conflicts of interest}

No potential conflicts of interest relevant to this article was reported.

\section{ORCID}

Jongmoo Park, https://orcid.org/0000-0002-7004-8128

Jae Won Park, https://orcid.org/0000-0003-1100-5301

Min Kyu Kang, https://orcid.org/0000-0002-7962-7054

\section{References}

1. Jung KW, Won YJ, Kong HJ, Lee ES; Community of Population-Based Regional Cancer Registries. Cancer statistics in Korea: incidence, mortality, survival, and prevalence in 2015. Cancer Res Treat 2018;50:303-16.

2. Perz JF, Armstrong GL, Farrington LA, Hutin YJ, Bell BP. The contributions of hepatitis $\mathrm{B}$ virus and hepatitis $\mathrm{C}$ virus infections to cirrhosis and primary liver cancer worldwide. J Hepatol 2006;45:529-38.

3. Ock M, Choi WJ, Jo MW. Trend analysis of major cancer statis- 
tics according to sex and severity levels in Korea. PLoS One 2018;13:e0203110

4. European Association for the Study of the Liver. EASL clinical practice guidelines: management of hepatocellular carcinoma. J Hepatol 2018;69:182-236.

5. Korean Liver Cancer Association; National Cancer Center. 2018 Korean Liver Cancer Association-National Cancer Center Korea Practice Guidelines for the management of hepatocellular Carcinoma. Gut Liver 2019;13:227-99.

6. Delis SG, Dervenis C. Selection criteria for liver resection in patients with hepatocellular carcinoma and chronic liver disease. World J Gastroenterol 2008;14:3452-60.

7. Tiong L, Maddern GJ. Systematic review and meta-analysis of survival and disease recurrence after radiofrequency ablation for hepatocellular carcinoma. BrJ Surg 2011;98:1210-24.

8. Benedict SH, Yenice KM, Followill D, Galvin JM, Hinson W, Kavanagh $B$, et al. Stereotactic body radiation therapy: the report of AAPM Task Group 101. Med Phys 2010;37:4078-101.

9. Rim CH, Kim HJ, Seong J. Clinical feasibility and efficacy of stereotactic body radiotherapy for hepatocellular carcinoma: a systematic review and meta-analysis of observational studies. Radiother Oncol 2019;131:135-44.

10. Vogel A, Cervantes A, Chau I, Daniele B, Llovet J, Meyer T, et al. Hepatocellular carcinoma: ESMO Clinical Practice Guidelines for diagnosis, treatment and follow-up. Ann Oncol 2018;29(Suppl 4):iv238-55.

11. Murray LJ, Dawson LA. Advances in stereotactic body radiation therapy for hepatocellular carcinoma. Semin Radiat Oncol 2017;27:247-55.

12. Yoon SM, Lim YS, Park MJ, Kim SY, Cho B, Shim JH, et al. Stereotactic body radiation therapy as an alternative treatment for small hepatocellular carcinoma. PLoS One 2013;8:e79854

13. Sanuki N, Takeda A, Oku Y, Mizuno T, Aoki Y, Eriguchi T, et al. Stereotactic body radiotherapy for small hepatocellular carcinoma: a retrospective outcome analysis in 185 patients. Acta Oncol 2014;53:399-404.

14. Sanuki N, Takeda A, Kunieda E. Role of stereotactic body radiation therapy for hepatocellular carcinoma. World J Gastroenterol 2014;20:3100-11.

15. Dawson LA, Brock KK, Kazanjian S, Fitch D, McGinn CJ, Lawrence TS, et al. The reproducibility of organ position using active breathing control $(\mathrm{ABC})$ during liver radiotherapy. Int $J$ Radiat Oncol Biol Phys 2001;51:1410-21.

16. Herfarth KK, Debus J, Lohr F, Bahner ML, Fritz P, Höss A, et al. Extracranial stereotactic radiation therapy: set-up accuracy of patients treated for liver metastases. Int J Radiat Oncol Biol Phys 2000;46:329-35.
17. Brock KK. Imaging and image-guided radiation therapy in liver cancer. Semin Radiat Oncol 2011;21:247-55.

18. Park SH, Kim JC, Kang MK. Technical advances in external radiotherapy for hepatocellular carcinoma. World J Gastroenterol 2016;22:7311-21.

19. Scorsetti M, Comito T, Cozzi L, Clerici E, Tozzi A, Franzese C, et al. The challenge of inoperable hepatocellular carcinoma (HCC): results of a single-institutional experience on stereotactic body radiation therapy (SBRT). J Cancer Res Clin Oncol 2015;141:1301-9.

20. Lee KH, Yu JI, Park HC, Park SY, Shin JS, Shin EH, et al. Is higher dose always the right answer in stereotactic body radiation therapy for small hepatocellular carcinoma? Radiat Oncol J 2018;36:129-38.

21. Jang WI, Kim MS, Bae SH, Cho CK, Yoo HJ, Seo YS, et al. High-dose stereotactic body radiotherapy correlates increased local control and overall survival in patients with inoperable hepatocellular carcinoma. Radiat Oncol 2013;8:250.

22. Mizumoto M, Okumura T, Hashimoto T, Fukuda K, Oshiro Y, Fukumitsu N, et al. Proton beam therapy for hepatocellular carcinoma: a comparison of three treatment protocols. Int J Radiat Oncol Biol Phys 2011;81:1039-45.

23. Kasuya G, Kato H, Yasuda S, Tsuji H, Yamada S, Haruyama Y, et al. Progressive hypofractionated carbon-ion radiotherapy for hepatocellular carcinoma: combined analyses of 2 prospective trials. Cancer 2017;123:3955-65.

24. Toramatsu C, Katoh N, Shimizu S, Nihongi H, Matsuura T, Takao S, et al. What is the appropriate size criterion for proton radiotherapy for hepatocellular carcinoma? A dosimetric comparison of spot-scanning proton therapy versus intensity-modulated radiation therapy. Radiat Oncol 2013;8:48.

25. Gandhi SJ, Liang X, Ding X, Zhu TC, Ben-Josef E, Plastaras JP, et al. Clinical decision tool for optimal delivery of liver stereotactic body radiation therapy: Photons versus protons. Pract Radiat Oncol 2015;5:209-18.

26. Blomgren H, Lax I, Näslund I, Svanström R. Stereotactic high dose fraction radiation therapy of extracranial tumors using an accelerator. Clinical experience of the first thirty-one patients. Acta Oncol 1995;34:861-70.

27. Shiozawa K, Watanabe M, Ikehara T, Matsukiyo Y, Kogame M, Kishimoto Y, et al. Comparison of percutaneous radiofrequency ablation and CyberKnife ${ }^{\circledast}$ for initial solitary hepatocellular carcinoma: A pilot study. World J Gastroenterol 2015;21:13490-9.

28. Wahl DR, Stenmark MH, Tao Y, Pollom EL, Caoili EM, Lawrence TS, et al. Outcomes after stereotactic body radiotherapy or radiofrequency ablation for hepatocellular carcinoma. J Clin Oncol 2016;34:452-9. 
29. Bujold A, Massey CA, Kim JJ, Brierley J, Cho C, Wong RK, et al. Sequential phase I and II trials of stereotactic body radiotherapy for locally advanced hepatocellular carcinoma. J Clin Oncol 2013;31:1631-9.

30. Rhim H, Yoon KH, Lee JM, Cho Y, Cho JS, Kim SH, et al. Major complications after radio-frequency thermal ablation of hepatic tumors: spectrum of imaging findings. Radiographics 2003;23:123-34.

31. Rice SL, Bale R, Breen DJ, de Baere T, Denys A, Guiu B, et al. The management of colorectal cancer liver metastases: the interventional radiology viewpoint. Int J Radiat Oncol Biol Phys 2019;103:537-9.

32. Lee S, Kim H, Ji Y, Cho B, Kim SS, Jung J, et al. Evaluation of hepatic toxicity after repeated stereotactic body radiation therapy for recurrent hepatocellular carcinoma using deformable image registration. Sci Rep 2018;8:16224.

33. Rajyaguru DJ, Borgert AJ, Smith AL, Thomes RM, Conway PD, Halfdanarson TR, et al. Radiofrequency ablation versus stereotactic body radiotherapy for localized hepatocellular carcinoma in nonsurgically managed patients: analysis of the national cancer database. J Clin Oncol 2018;36:600-8.

34. Nakano R, Ohira M, Kobayashi T, Ide K, Tahara H, Kuroda S, et al. Hepatectomy versus stereotactic body radiotherapy for primary early hepatocellular carcinoma: a propensity-matched analysis in a single institution. Surgery 2018;164:219-26.

35. Su TS, Liang P, Liang J, Lu HZ, Jiang HY, Cheng T, et al. Longterm survival analysis of stereotactic ablative radiotherapy versus liver resection for small hepatocellular carcinoma. Int J Radiat Oncol Biol Phys 2017;98:639-46.

36. Pan CC, Kavanagh BD, Dawson LA, Li XA, Das SK, Miften M, et al. Radiation-associated liver injury. Int J Radiat Oncol Biol Phys 2010;76(3 Suppl):S94-100.

37. Liang SX, Zhu XD, Xu ZY, Zhu J, Zhao JD, Lu HJ, et al. Radiation-induced liver disease in three-dimensional conformal radiation therapy for primary liver carcinoma: the risk factors and hepatic radiation tolerance. Int J Radiat Oncol Biol Phys 2006;65:426-34.

38. Jung J, Yoon SM, Kim SY, Cho B, Park JH, Kim SS, et al. Radiation-induced liver disease after stereotactic body radiotherapy for small hepatocellular carcinoma: clinical and dose-volumetric parameters. Radiat Oncol 2013;8:249.

39. Culleton S, Jiang H, Haddad CR, Kim J, Brierley J, Brade A, et al. Outcomes following definitive stereotactic body radiotherapy for patients with Child-Pugh B or C hepatocellular carcinoma. Radiother Oncol 2014;111:412-7.

40. Su TS, Liang P, Lu HZ, Liang J, Gao YC, Zhou Y, et al. Stereotactic body radiation therapy for small primary or recurrent hepa- tocellular carcinoma in 132 Chinese patients. J Surg Oncol 2016;113:181-7.

41. Dawson LA. Overview: where does radiation therapy fit in the spectrum of liver cancer local-regional therapies? Semin Radiat Oncol 2011;21:241-6.

42. Kavanagh BD, Pan CC, Dawson LA, Das SK, Li XA, Ten Haken $\mathrm{RK}$, et al. Radiation dose-volume effects in the stomach and small bowel. Int J Radiat Oncol Biol Phys 2010;76(3 Suppl):S101-7.

43. Bae SH, Kim MS, Kim SY, Jang WI, Cho CK, Yoo HJ, et al. Severe intestinal toxicity after stereotactic ablative radiotherapy for abdominopelvic malignancies. Int J Colorectal Dis 2013; 28:1707-13.

44. LaCouture TA, Xue J, Subedi G, Xu Q, Lee JT, Kubicek G, et al. Small bowel dose tolerance for stereotactic body radiation therapy. Semin Radiat Oncol 2016;26:157-64.

45. Goldsmith C, Price P, Cross T, Loughlin S, Cowley I, Plowman N. Dose-volume histogram analysis of stereotactic body radiotherapy treatment of pancreatic cancer: a focus on duodenal dose constraints. Semin Radiat Oncol 2016;26:149-56.

46. Yamashita H, Onishi H, Murakami N, Matsumoto Y, Matsuo Y, Nomiya T, et al. Survival outcomes after stereotactic body radiotherapy for 79 Japanese patients with hepatocellular carcinoma. J Radiat Res 2015;56:561-7.

47. Sapir E, Tao Y, Schipper MJ, Bazzi L, Novelli PM, Devlin P, et al. Stereotactic body radiation therapy as an alternative to transarterial chemoembolization for hepatocellular carcinoma. Int J Radiat Oncol Biol Phys 2018;100:122-30.

48. Jeong Y, Jung J, Cho B, Kwak J, Jeong C, Kim JH, et al. Stereotactic body radiation therapy using a respiratory-gated volumetric-modulated arc therapy technique for small hepatocellular carcinoma. BMC Cancer 2018; 18:416.

49. Eriguchi T, Takeda A, Sanuki N, Oku Y, Aoki Y, Shigematsu N, et al. Acceptable toxicity after stereotactic body radiation therapy for liver tumors adjacent to the central biliary system. Int J Radiat Oncol Biol Phys 2013;85:1006-11.

50. Toesca DA, Osmundson EC, Eyben RV, Shaffer JL, Lu P, Koong AC, et al. Central liver toxicity after SBRT: an expanded analysis and predictive nomogram. Radiother Oncol 2017;122:130-6.

51. O'Connor JK, Trotter J, Davis GL, Dempster J, Klintmalm GB, Goldstein RM. Long-term outcomes of stereotactic body radiation therapy in the treatment of hepatocellular cancer as a bridge to transplantation. Liver Transpl 2012;18:949-54.

52. Andolino DL, Johnson CS, Maluccio M, Kwo P, Tector AJ, Zook J, et al. Stereotactic body radiotherapy for primary hepatocellular carcinoma. Int J Radiat Oncol Biol Phys 2011;81:e44753. 
53. Sapisochin G, Barry A, Doherty M, Fischer S, Goldaracena N, Rosales R, et al. Stereotactic body radiotherapy vs. TACE or RFA as a bridge to transplant in patients with hepatocellular carcinoma. An intention-to-treat analysis. J Hepatol 2017;67:92-9.

54. Mohamed M, Katz AW, Tejani MA, Sharma AK, Kashyap R, Noel MS, et al. Comparison of outcomes between SBRT, yttrium-90 radioembolization, transarterial chemoembolization, and radiofrequency ablation as bridge to transplant for hepatocellular carcinoma. Adv Radiat Oncol 2015;1:35-42.

55. Kim JY, Chung SM, Choi BO, Kay CS. Hepatocellular carcinoma with portal vein tumor thrombosis: Improved treatment outcomes with external beam radiation therapy. Hepatol Res 2011;41:813-24.

56. Im JH, Yoon SM, Park HC, Kim JH, Yu JI, Kim TH, et al. Radiotherapeutic strategies for hepatocellular carcinoma with portal vein tumour thrombosis in a hepatitis B endemic area. Liver Int 2017;37:90-100.

57. Bae SH, Kim MS, Jang WI, Kim JH, Kim WC, Kim JH, et al. Multicenter planning comparison of stereotactic body radiotherapy for hepatocellular carcinoma with major portal vein tumor thrombosis (KROG 16-17). J Liver Cancer 2018;18:130 41.

58. Xi M, Zhang L, Zhao L, Li QQ, Guo SP, Feng ZZ, et al. Effectiveness of stereotactic body radiotherapy for hepatocellular carcinoma with portal vein and/or inferior vena cava tumor thrombosis. PLoS One 2013;8:e63864

59. Kang J, Nie Q, DU R, Zhang L, Zhang J, Li Q et al. Stereotactic body radiotherapy combined with transarterial chemoembolization for hepatocellular carcinoma with portal vein tumor thrombosis. Mol Clin Oncol 2014;2:43-50.

60. Shui Y, Yu W, Ren X, Guo Y, Xu J, Ma T, et al. Stereotactic body radiotherapy based treatment for hepatocellular carcinoma with extensive portal vein tumor thrombosis. Radiat Oncol 2018; 13:188.

61. Meng MB, Cui YL, Lu Y, She B, Chen Y, Guan YS, et al. Transcatheter arterial chemoembolization in combination with radiotherapy for unresectable hepatocellular carcinoma: a systematic review and meta-analysis. Radiother Oncol 2009;92:18494.

62. Jacob R, Turley F, Redden DT, Saddekni S, Aal AK, Keene K, et al. Adjuvant stereotactic body radiotherapy following transarterial chemoembolization in patients with non-resectable hepatocellular carcinoma tumours of $\geq 3 \mathrm{~cm}$. HPB (Oxford) 2015;17:140-9.

63. PaikEK, Kim MS, Jang WI, Seo YS, Cho CK, Yoo HJ, et al. Benefits of stereotactic ablative radiotherapy combined with incomplete transcatheter arterial chemoembolization in hepatocellular carcinoma. Radiat Oncol 2016;11:22.

64. Llovet JM, Ricci S, Mazzaferro V, Hilgard P, Gane E, Blanc JF, et al. Sorafenib in advanced hepatocellular carcinoma. N Engl J Med 2008;359:378-90.

65. Yu W, Gu K, Yu Z, Yuan D, He M, Ma N, et al. Sorafenib potentiates irradiation effect in hepatocellular carcinoma in vitro and in vivo. Cancer Lett 2013;329:109-17.

66. Barney BM, Markovic SN, Laack NN, Miller RC, Sarkaria JN, Macdonald OK, et al. Increased bowel toxicity in patients treated with a vascular endothelial growth factor inhibitor (VEGFI) after stereotactic body radiation therapy (SBRT). Int J Radiat Oncol Biol Phys 2013;87:73-80.

67. Brade AM, Ng S, Brierley J, Kim J, Dinniwell R, Ringash J, et al. Phase 1 trial of sorafenib and stereotactic body radiation therapy for hepatocellular carcinoma. Int J Radiat Oncol Biol Phys 2016;94:580-7.

68. Kang J, Demaria S, Formenti S. Current clinical trials testing the combination of immunotherapy with radiotherapy. J Immunother Cancer 2016;4:51.

69. Bernstein MB, Krishnan S, Hodge JW, Chang JY. Immunotherapy and stereotactic ablative radiotherapy (ISABR): a curative approach? Nat Rev Clin Oncol 2016;13:516-24.

70. Formenti SC. Optimizing dose per fraction: a new chapter in the story of the abscopal effect? Int J Radiat Oncol Biol Phys 2017;99:677-9.

71. Vanpouille-Box C, Formenti SC, Demaria S. Toward precision radiotherapy for use with immune checkpoint blockers. Clin Cancer Res 2018;24:259-65.

72. Kim KJ, Kim JH, Lee SJ, Lee EJ, Shin EC, Seong J. Radiation improves antitumor effect of immune checkpoint inhibitor in murine hepatocellular carcinoma model. Oncotarget 2017;8: 41242-55. 